\title{
The optical counterpart of IGR J00291+5934 in quiescence ${ }^{\star}$
}

\author{
P. D’Avanzo ${ }^{1,2}$, S. Campana ${ }^{1}$, S. Covino ${ }^{1}$, G. L. Israel ${ }^{3}$, L. Stella $^{3}$, and G. Andreuzzi ${ }^{4}$ \\ 1 INAF - Osservatorio Astronomico di Brera, via E. Bianchi 46, 23807 Merate (Lc), Italy \\ e-mail: paolo.davanzo@brera.inaf.it \\ 2 Università degli Studi dell'Insubria, Dipartimento di Fisica e Matematica, via Valleggio 11, 22100 Como, Italy \\ INAF - Osservatorio Astronomico di Roma, via Frascati 33, 00040 Monte Porzio Catone, Roma, Italy \\ ${ }^{4}$ Fundación Galileo Galilei - INAF, Fundación Canaria, C. Alvarez de Abreu, 70, 38700 S/C. de La Palma, Spain
}

Received 2 March 2007 / Accepted 18 July 2007

ABSTRACT

\begin{abstract}
Aims. The recent (December 2004) discovery of the sixth accretion-powered millisecond X-ray pulsar IGR J00291+5934 provides a very good chance to deepen our knowledge of such systems. Although these systems are well studied at high energies, poor informations are available for their optical/NIR counterparts during quiescence. Up to now, only for SAX J1808.4-3658, the first discovered system of this type, we have a secure multiband detection of its optical counterpart in quiescence. Among the seven known system IGR J00291+5934 is the one that resembles SAX J1808.4-3658 more closely.

Methods. With the Italian $3.6 \mathrm{~m}$ TNG telescope, we have performed deep optical and NIR photometry of the field of IGR J00291+5934 during quiescence in order to look for the presence of a variable counterpart.

Results. We present here the first multiband $(V R I J H)$ detection of the optical and NIR counterpart of IGR J00291+5934 in quiescence as well as a deep upper limit in the $K$-band. We obtain an optical light curve that shows variability consistent with a sinusoidal modulation at the known $2.46 \mathrm{~h}$ orbital period and present evidence for a strongly irradiated companion.
\end{abstract}

Key words. accretion, accretion disks - X-rays: binaries - stars: neutron

\section{Introduction}

In 1998, the discovery of the first accretion-powered millisecond X-ray pulsar SAX J1808.4-3658 (Wijnands \& van der Klis 1998; Chakrabarthy \& Morgan 1998), confirmed the evolutionary link between Low Mass X-Ray Binaries (LMXBs) and millisecond radio pulsars (see e.g. Bhattacharya \& van den Heuvel 1991; Tauris \& van den Heuvel 2004) supporting the idea that the formers are the progenitors of the latters. In the following years, six more accretionpowered millisecond X-ray pulsars have been discovered: XTE J1751-305 (Markwardt et al. 2002), XTE J0929-314 (Galloway et al. 2002), XTE J1807-294 (Markwardt et al. 2003; Campana et al. 2003), XTE J1814-314 (Markwardt \& Swank 2003; Strohmayer et al. 2003), IGR J00291+5934 (Galloway et al. 2005) and HETE J1900.1-2455 (Morgan et al. 2005; Campana 2005). All these systems are transients of the Soft X-Ray Transients class (SXRTs, for a review see Campana et al. 1998), have orbital periods in the range between $40 \mathrm{~min}$ and $4.5 \mathrm{~h}$ and spin frequencies from 1.7 to $5.4 \mathrm{~ms}$. These seven accreting millisecond pulsars are well studied at high energies, especially in the X-rays, both in outburst and in quiescence (see Wijnands 2005 , for a review). On the other hand, with the significant exception of SAX J1808.4-3658, their optical/NIR quiescent counterparts are only poorly known. The optical light curve of SAX J1808.4-3658 in outburst and quiescence shows variability modulated at the orbital period, in antiphase with the X-ray light curve (Homer et al. 2001; Campana et al. 2004). This is unlike

* Based on observations made with the Italian Telescopio Nazionale Galileo (TNG) operated on the island of La Palma by the Fundación Galileo Galilei of the INAF (Istituto Nazionale di Astrofisica) at the Spanish Observatorio del Roque de los Muchachos of the Instituto de Astrofisica de Canarias. other quiescent transient that normally show a double-humped morphology, due to an ellipsoidal modulation, and indicates that the companion star is subject to some irradiation. Burderi et al. (2003) proposed that the irradiation is due to the release of rotational energy by the fast spinning neutron star, switched on, as a radio pulsar, during quiescence. Following this idea, Campana et al. (2004) measured the required irradiating luminosity needed to match the optical flux and found that it is a factor of about 100 larger than the quiescent X-ray luminosity of the system. Neither accretion-driven X-rays nor the intrinsic luminosity of the secondary star or the disc can account for it. So, these authors conclude that the only source of energy available within the system is the rotational energy of the neutron star, reactivated as a millisecond radio pulsar. Optical and NIR observations performed in the past by different groups for the other systems of this class only led to deep upper limits for the counterparts of XTE J1751-305 (Jonker et al. 2003) and XTE J1814-314 (Krauss et al. 2005) or to the detection of very faint candidates, if any (Monelli et al. 2005, for XTE J0929-314). The intrinsic faintness of the targets, in combination with the high interstellar absorption and high stellar crowding of the relevant fields are among the main reasons for the lack of detections at optical wavelengths.

\section{IGR J00291+5934}

IGR J00291+5934 was discovered in outburst on December 2nd 2004 during a routine monitoring of the Galactic plane with the INTEGRAL satellite (Eckert et al. 2004). Follow-up RXTE observations established the source as a $598.88 \mathrm{~Hz}(1.67 \mathrm{~ms})$ accretion-powered X-ray pulsar (Markwardt et al. 2004a) with a $2.46 \mathrm{~h}$ orbital period 
(Markwardt et al. 2004b). With a spin frequency of $599 \mathrm{~Hz}$, IGR J00291+5934 is the fastest known accretion-powered millisecond pulsar. An $R \sim 17.4$ candidate optical counterpart was identified within the INTEGRAL error circle (Fox \& Kulkarni 2004). Optical spectroscopy of the candidate revealed weak $\mathrm{He}$ II and $\mathrm{H} \alpha$ emission, supporting its association with IGR J00291+5934 (Roelofs et al. 2004). The mass function gives an upper limit of $0.16 M_{\odot}$ for the mass of the companion star indicating that, like SAX J1808.4-3658, the secondary is probably a hot brown dwarf (Galloway et al. 2005). Radio observations provided evidence for variable emission, consistent with the counterpart position (Pooley 2004; Fender et al. 2004). Signs of source activity during two occasions in the past were found in the RXTE/ASM light curves, with a probable recurrence of about 3 years (Remillard 2004). Observations made with INTEGRAL and RXTE during outburst revealed a power-law spectrum (with photon index 1.7-1.8) consistent with a column density of $N_{\mathrm{H}} \sim 10^{22} \mathrm{~cm}^{-2}$ (Shaw et al. 2005; Galloway et al. 2005). Paizis et al. (2005) performed Chandra and RXTE spectroscopy during outburst and modeled the spectra with a combination of a thermal component (probably due to a hot-spot on the neutron star's surface) and a power-law with a hydrogen column of $N_{\mathrm{H}}=4.3 \times 10^{21} \mathrm{~cm}^{-2}$. In quiescence IGR J00291+5934 has been recently detected by Chandra (and historically ROSAT; Jonker et al. 2005). The source quiescent $0.5-10 \mathrm{keV}$ unabsorbed flux was $\sim 8 \times 10^{-14} \mathrm{erg} \mathrm{cm}^{-2} \mathrm{~s}^{-1}$ (with $N_{\mathrm{H}}$ fixed to $2.8 \times 10^{21} \mathrm{~cm}^{-2}$ ) during two Chandra observations following the 2004 outburst. However, in the third observation a few weeks later, IGR J00291+5934 showed a flux a factor of two higher. This yielded the first clear evidence for variability in the quiescent X-ray emission of an accretion-powered millisecond X-ray pulsar. Subsequent quiescent Chandra observations carried out in November 2005 showed a power-law spectrum (with photon index 2.0-2.9) and unabsorbed 0.5-10 keV flux of $\sim 7 \times 10^{-14} \mathrm{erg} \mathrm{cm} \mathrm{cm}^{-2} \mathrm{~s}^{-1}$ for $N_{\mathrm{H}}=4.6 \times 10^{21} \mathrm{~cm}^{-2}$. Very recently, Torres et al. (2007) reported about optical, NIR and Chandra observations of IGR J00291+5934 carried out during outburst as well as in quiescence. They model the quiescent 0.5-10 keV spectrum with a power-law with a photon index of $\sim 2.4$ and $N_{\mathrm{H}}=(4-5) \times 10^{21} \mathrm{~cm}^{-2}$.

The non-detection of X-ray bursts (Galloway et al. 2005) makes the estimate of the distance of IGR J00291+5934 difficult. Shaw et al. (2005) give an upper limit of $3.3 \mathrm{kpc}$ considering source position with respect to the Galactic Centre and the measured absorbing column density ${ }^{1}$. From the INTEGRAL observed total fluence and taking into account the recurrence time of 3 years, Galloway et al. (2005) and Falanga et al. (2005) find a minimum distance of, respectively, 4 and $4.7 \mathrm{kpc}$. By considering the non-detection of thermonuclear burst, Galloway et al. (2005) suggest that the source distance should not be significantly greater than $4 \mathrm{kpc}$. Jonker et al. (2005) estimate the source distance to be between $2.6-3.6 \mathrm{kpc}$ assuming a quiescent X-ray luminosity of $(5-10) \times 10^{31} \mathrm{erg} \mathrm{s}^{-1}$, similar to that of SAX J1808.4-3658 and XTE J0929-314 (Campana et al. 2002; Wijnands et al. 2005). Torres et al. (2007) estimated a distance of 2-4 kpc based on an estimate of the critical X-ray luminosity necessary to ionize the accretion disc and produce the observed

\footnotetext{
1 Those authors used $N_{\mathrm{H}}=(2.8 \pm 0.4) \times 10^{21} \mathrm{~cm}^{-2}$ as measured by Nowak et al. (2004) with Chandra. Even if this value has been largely used in the literature, we note that from a subsequent analysis of the same Chandra data, the same group measured a value of $N_{\mathrm{H}}=(4.3 \pm$ $0.4) \times 10^{21} \mathrm{~cm}^{-2}$ (as reported in Paizis et al. 2005). Using this refined value of $N_{\mathrm{H}}$ and with the same arguments of Shaw et al. (2005) we derive for the distance of IGR J00291+5934 an upper limit of $\sim 6.0 \mathrm{kpc}$.
}

Table 1. Observation log for IGR J00291+5934.

\begin{tabular}{ccccc}
\hline \hline $\begin{array}{c}\text { UT observation } \\
\text { (YYYmmdd) }\end{array}$ & $\begin{array}{c}\text { Exposure } \\
(\mathrm{s})\end{array}$ & $\begin{array}{c}\text { Seeing } \\
(\operatorname{arcsec})\end{array}$ & Instrument & Filter \\
\hline 20050811.06237 & $23 \times 260 \mathrm{~s}$ & $1.4^{\prime \prime}$ & TNG/LSR & $V$ \\
20050811.17013 & $24 \times 260 \mathrm{~s}$ & $1.2^{\prime \prime}$ & TNG/LSR & $R$ \\
20051103.07361 & $23 \times 260 \mathrm{~s}$ & $0.9^{\prime \prime}$ & TNG/LSR & $I$ \\
20050902.04549 & $30 \times 3 \times 20 \mathrm{~s}$ & $0.7^{\prime \prime}$ & TNG/NICS & $J$ \\
20050902.06426 & $20 \times 3 \times 20 \mathrm{~s}$ & $0.7^{\prime \prime}$ & TNG/NICS & $H$ \\
20050902.06983 & $20 \times 3 \times 20 \mathrm{~s}$ & $0.8^{\prime \prime}$ & TNG/NICS & $K$ \\
\hline
\end{tabular}

X-ray light curve during outburst. Finally, Burderi et al. (2006) derive the mass accretion rate from timing of the source and estimate a bolometric luminosity of about $10^{38} \mathrm{erg} \mathrm{s}^{-1}$ concluding that the source is in a distance range of $7-10 \mathrm{kpc}$. They also note that $10 \mathrm{kpc}$ is close to the edge of our Galaxy in the direction of IGR J00291+5934. In the following, we assume a fiducial distance of $3 \mathrm{kpc}$.

\section{Observations and data reduction}

Optical and NIR observations of the field of IGR J00291+5934 were carried out with the Italian 3.6-m TNG telescope, sited in Canary Island, in 2005 on three different nights. Optical observations were made using the DOLORES (Device Optimized for the LOw RESolution) camera, installed at the Nasmyth-B focus of the TNG. The detector is a Loral thinned and back-illuminated $2048 \times 2048$ CCD. The scale is 0.275 arcsec/pix which yields a field of view of about $9.4 \times 9.4$ arcmin. A set of $V$ and $R$-band images was taken on 2005 August 11 and a set of $I$-band images was taken on November 3 . The exposure time of each frame was $260 \mathrm{~s}$, with a detector dead time of about $70 \mathrm{~s}$. With each optical filter we covered about $80-90 \%$ of the $2.46 \mathrm{~h}$ orbital period, with approximately a 0.04 phase resolution. NIR observations were performed on 2005 September 2nd using the NICS (Near Infrared Camera Spectrometer) infrared camera, which is based on a $\mathrm{HgCdTe} H a w a i i 1024 \times 1024$ array $(4.2 \times 4.2$ arcmin $)$. The instrument is installed at the Nasmyth-A focus of the TNG. We cycled through $J H K$ filters with exposures times of 20 and $30 \mathrm{~s}$, obtaining a total exposure of about $30 \mathrm{~min}$ for each filter. The complete observing log is presented in Table 1

Image reduction was carried out by following the standard procedures: subtraction of an averaged bias frame, division by a normalized flat frame. Astrometry was performed using the USNOB $1.0^{2}$ and the 2 MASS $^{3}$ catalogues. PSF-photometry was made with the ESO-MIDAS ${ }^{4}$ daophot task for all the objects in the field. The calibration was done against Landolt standard stars for $V$ and $R$ filters and against the 2MASS catalog for NIR filters. I-band observations were carried out under nonphotometric conditions, so we could not calibrate them using Landolt standard stars as reference. We thus used the USNOB1.0 catalog to calibrate the $I$ frames exploiting, to this end, a large sample of local isolated and non-saturated field stars. This led to a slight increase in the uncertainty of the magnitude measurement, even if $I$-band images were taken under good seeing conditions (see Table 1).

In order to minimize any systematic effect, we performed differential photometry with respect to a selection of local isolated and non-saturated standard stars. In addition to ordinary photometry, we also carried out image subtraction with the ISIS

\footnotetext{
2 http://www.nofs.navy.mil/data/fchpix/

3 http://www.ipac. caltech.edu/2mass/

4 http://www . eso.org/projects/esomidas/
} 


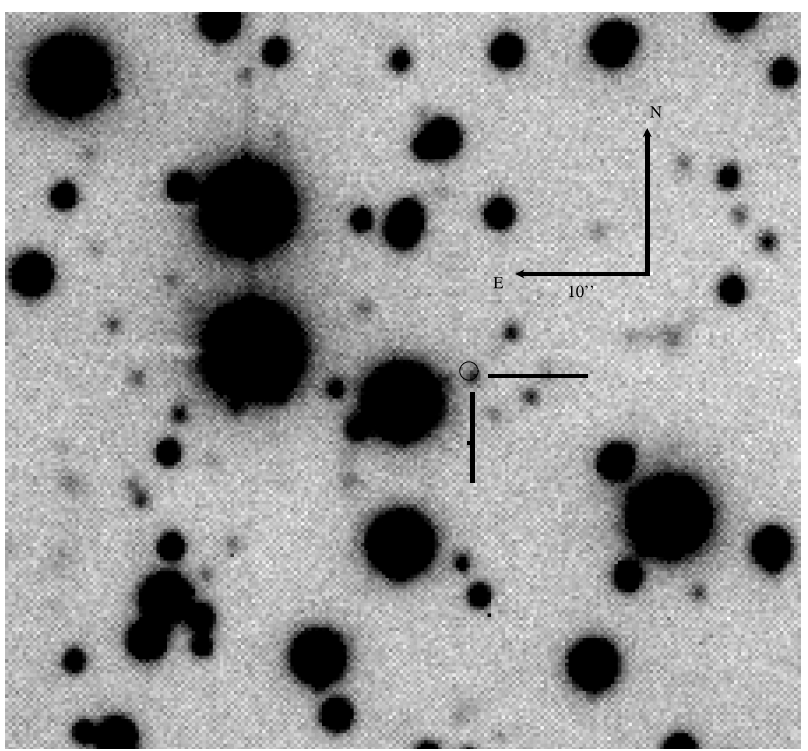

Fig. 1. $R$-band finding chart for IGR J00291+5934. The source is indicated by solid bars. The black circle represents the Chandra error box.

package (Alard 2000; Alard \& Lupton 1998) in order to check for variability of the sources in the field.

\section{Results}

Our entire dataset consist of about 20-30 frames for each filter (see in Table 1). To increase the signal to noise ratio, we computed an average of all our images for each photometric band. In all our $V, R$ and $I$ averaged frames we clearly detect an object inside the 0.6 arcsec radius Chandra error box (Paizis et al. 2005), while in our $J$ and $H$-band averaged frame we only have a marginal detection for this object. A finding chart is reported in Fig. 1. The position of the detected source is RA = 00:29:03.07, Dec $=+59: 34: 19.12(\mathrm{~J} 2000)$ with an uncertainty of $0.4^{\prime \prime}$. This position is coincident with the one of the optical counterpart of IGR J00291+5934 detected by Fox \& Kulkarni (2004) during the December 2004 outburst (with an error of $0.5^{\prime \prime}$ ) and by Torres et al. (2007) during both outburst and quiescence (with an error of $0.05^{\prime \prime}$ ) and consistent with the radio position reported by Rupen et al. (2004), with an uncertainty of less than $0.1^{\prime \prime}$. Results of PSF-photometry for this source in our optical and NIR averaged frames are reported in Table 2 . We note that our $R$-band magnitude value, as expected, is consistent with the one reported by Torres et al. (2007) measured during quiescence, in late 2005 (about two months after our observations). On the other hand, as reported in Table 2, we did not detect any NIR counterpart of IGR J00291+5934 in $K$-band down to a limiting value of $19.3 \mathrm{mag}$ ( $3 \sigma$ confidence level). We note that Torres et al. (2007) reported a detection at $K=19.0 \pm 0.1$ for the 2005 Jan. 24 observation, about fifty days after the discovery of the source in outburst and about twenty days after its return to quiescence (Jonker et al. 2005). A possible explanation of this discrepancy is that the $K$-band observations of Torres et al. (2007) took place when the source was in the tail of the outburst, and quiescence had not been reached yet.

Once we identified the candidate, we searched for variability given that, as reported in Sect. 3, we cover about 80-90\% of the orbital period with each optical filter. As a first check, we performed image subtraction with the ISIS package (Alard 2000; Alard \& Lupton 1998) on our I-band images coadded into seven
Table 2. Results of photometry of IGR J00291+5934, all the values are uncorrected for reddening. In column five are reported the dereddening parameters used to correct our optical/NIR photometry of computed assuming $E(B-V)=0.74 \pm 0.07$ mag (see Sect. 5 for details).

\begin{tabular}{ccccc}
\hline \hline Filter & $\begin{array}{c}\lambda_{\mathrm{c}} \\
(\AA)\end{array}$ & $\begin{array}{c}\text { Total Exposure } \\
(\mathrm{s})\end{array}$ & Magnitude & $\begin{array}{c}\mathrm{A}_{\lambda} \\
(\mathrm{mag})\end{array}$ \\
\hline$V$ & 5270 & 5980 & $24.0 \pm 0.1$ & 2.39 \\
$R$ & 6440 & 6240 & $23.2 \pm 0.1$ & 1.79 \\
$I$ & 7980 & 5980 & $22.4 \pm 0.2$ & 1.28 \\
$J$ & 12700 & 1800 & $21.4 \pm 0.3$ & 0.58 \\
$H$ & 16300 & 1500 & $20.4 \pm 0.2$ & 0.39 \\
$K$ & 22000 & 1500 & $\geq 19.3(3 \sigma)$ & 0.25 \\
\hline
\end{tabular}

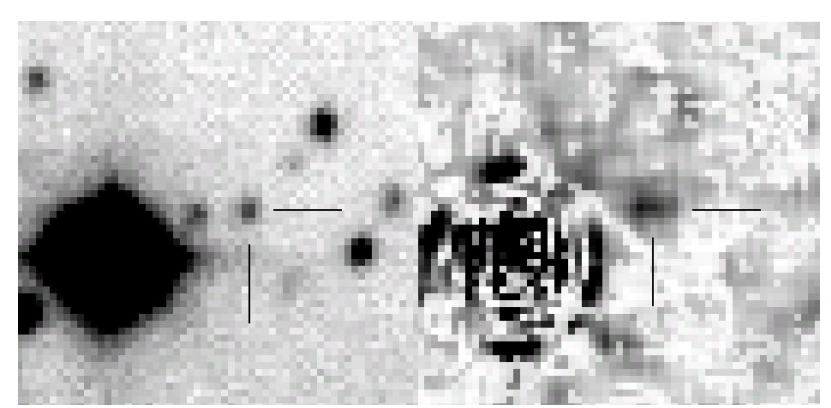

Fig. 2. $I$-band image subtraction for IGR J00291+5934. Left image shows the field of IGR J00291+5934, with its optical counterpart marked. Right image shows the result of the subtraction. A clear residual is present at the position of our source.

bins. We chose the $I$-band frames because they were those taken under better seeing conditions. The ISIS subtraction routine accounts for variation in the stellar PSF. A "reference frame" (in our case an average of two images taken at orbital phases 0.11 and 0.15 ) is subtracted to all the available images and photometry is performed on the residual images. For any variable object in the field the variation in flux, with respect to the reference frame, is given as output. Performing photometry on the reference frame, it is possible to calibrate in magnitudes the flux variations. The result of the image subtraction analysis is that our candidate was variable (Figs. 2, 3), with an indication of a sinusoidal modulation of semiamplitude $0.28 \pm 0.17 \mathrm{mag}(68 \%$ confidence level) at the $2.46 \mathrm{~h}$ orbital period (Fig. 3). The light curve shows a single minimum at phase 0 , i.e. at superior conjunction (when the neutron star is behind the companion) and a maximum at phase 0.5 (based on the X-ray ephemerides of Galloway et al. 2005). However, an F-test gives a probability of $94.2 \%$ with respect to a constant (period and phase constrained) so this represents only a marginal indication of variability of the source. In light of this, to improve our light curve, we tried to perform a more accurate phase-resolved photometry of our candidate in all our optical bands, where we have a clearer detection than in the NIR filters. Unfortunately, in none of the single optical frames our target was detected with signal-to-noise ratio high enough to look for variability with PSF-photometry and, considering the uncertainties in the measure of the magnitudes, we can only put a rough upper limit of about $0.3 \mathrm{mag}$ on the amplitude of variations in the individual bands. So, in order to improve the signal-to-noise ratio, we coadded our $R$ and $I$-band frames into six phase bins, with a mean 0.05 phase resolution and with all bins containing the same number of $R$ and $I$ band frames, in order to avoid color-related effects. We discarded $V$-band images because they were taken under worse seeing conditions. For each resulting bin we performed PSF-photometry of our source and of 


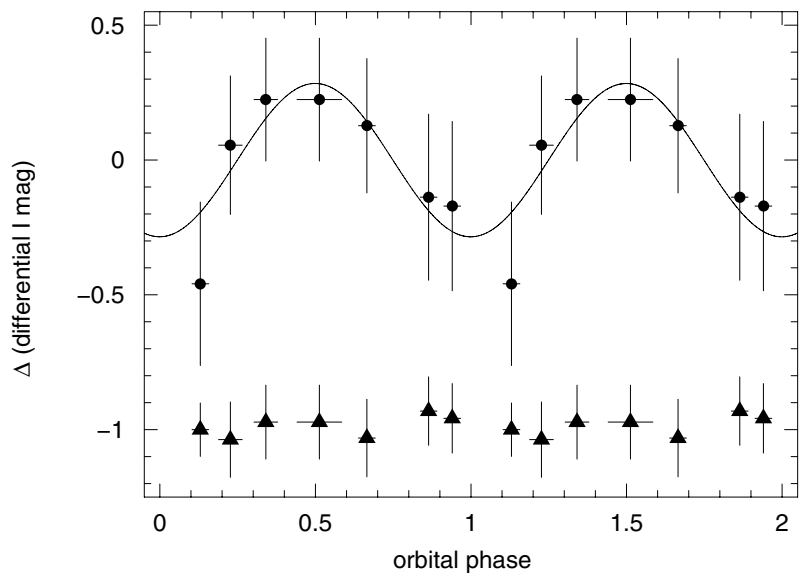

Fig. 3. I-band light curve of IGR J00291+5934 (dots) obtained with image subtraction technique (see Sect. 4 for details) in the phase range $0.10-0.97$. Two orbital phases are shown for clarity. The best sine-wave fit is also shown. The light curve for a field star of comparable brightness is plotted offset below (triangles).

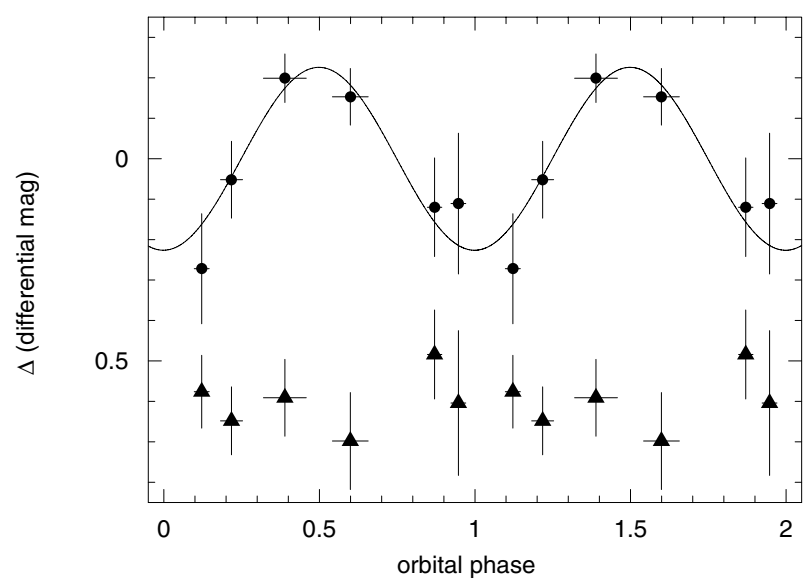

Fig. 4. Light curve of IGR J00291+5934 (dots) obtained with PSFphotometry of a set of $R$ and $I$ band frames coadded into six phase bins in the phase range 0.10-0.97. Two orbital phases are shown for clarity, the best sine-wave fit is also shown. The light curve for a field star of comparable brightness is plotted offset below (triangles).

a selected sample of bright, non-saturated, isolated stars assumed to be non-variable. The result of such differential photometry of the coadded $R$ and $I$-band frames confirms the variability of the source. Assuming that the observed variability could be related to orbital variations, we obtained a good agreement by fitting our data with a sinusoid of period $2.46 \mathrm{~h}$ (an F-test gives a probability of $99.8 \%$ with respect to a constant, period and phase constrained). The resulting semiamplitude is $0.22 \pm 0.09$ mag $(68 \%$ confidence level, see Fig. 4). This unambiguously identifies this source as the optical counterpart of IGR J00291+5934 and represents the first detection at both optical and NIR wavelengths of this source during quiescence. Further phase resolved photometry with larger telescopes is necessary to study the variability of the source in different bands.

\section{Discussion}

Based on X-ray pulses arrival times, Galloway et al. (2005) computed for IGR J00291+5934 a mass function of $2.8 \times 10^{-5} M_{\odot}$. This value can be combined with a system inclination $\leq 85^{\circ}$, derived from the lack of X-ray eclipses or dips, and implies a minimum mass for the secondary star of $0.04 M_{\odot}$. Assuming that the companion fills its Roche lobe and considering an isotropic, random distribution of inclinations, the companion star must have a mass $\leq 0.16 M_{\odot}$. Such mass limits imply that the mass donor in IGR J00291+5934 is likely a brown dwarf heated by $\mathrm{X}$-ray quiescent emission of the compact object (Galloway et al. 2005). A more tight constrain on the donor mass has recently been derived by Torres et al. (2007). Those authors measured the peak-to-peak separation of the $\mathrm{H} \alpha$ emission profile in the optical spectrum taken during outburst and use this value to estimate the inclination of the system. They estimate a range of inclination $22^{\circ} \leq i \leq 32^{\circ}$ which implies a mass for the companion star of $M_{\mathrm{c}}=0.04-0.11(0.09-0.13) M_{\odot}$ assuming a neutron star of $M_{\mathrm{X}}=1.4$ (2.0) $M_{\odot}$. Information about the companion's mass can also be obtained from our color photometry. To this end, we computed the unabsorbed $(V-R)=0.2 \pm 0.1,(V-J)=0.8 \pm$ $0.3,(V-H)=1.6 \pm 0.2,(R-J)=0.6 \pm 0.3$ and $(R-H)=1.4 \pm$ 0.2 colors and compared them with the theoretical mass-color diagrams computed for solar metallicity low-mass stars reported in Baraffe et al. (1998). The result is that the secondary's mass should be $M_{\mathrm{c}} \geq 0.8 M_{\odot}(2 \sigma$ c.l. $)$. Using the X-ray mass function (Galloway et al. 2005), we can derive for these masses the inclination of the system $i \leq 3^{\circ}$. The inferred inclination would thus be very low, and therefore highly improbable. A similar measure is reported by Torres et al. (2007) from the unabsorbed $(R-K)$ color of IGR J00291+5934.

The discrepancies in the estimate of the companion's mass can find an explanation under the hypothesis that the secondary star is subjected to irradiation from the compact object, as suggested by the optical light curve. Since during our observations IGR J00291+5934 was in quiescence, we try to investigate the possible causes of its optical/NIR emission. Assuming for the source a distance of $3 \mathrm{kpc}$ (as discussed in Sect. 2), then from an observed unabsorbed X-ray flux of $7 \times 10^{-14} \mathrm{erg} \mathrm{s}^{-1} \mathrm{~cm}^{-2}$ measured during quiescence with Chandra (Torres et al. 2007), we can derive a quiescent X-ray luminosity of $L_{X} \sim 8 \times$ $10^{31} \mathrm{erg} \mathrm{s}^{-1}$. A broadband spectrum, from optical to NIR, of IGR J00291+5934 can be obtained from our multiwavelength photometry. To compute the spectral energy distribution of the source we first need to correct our magnitudes for interstellar absorption computed with the relation $N(\mathrm{H}) / E(B-V)=5.8 \times$ $10^{21} \mathrm{~cm}^{-2} \mathrm{mag}^{-1}$ (Bohlin et al. 1979) and assuming $N(\mathrm{H})=$ $(4.3 \pm 0.4) \times 10^{21} \mathrm{~cm}^{-2}$ (Paizis et al. 2005). The resulting color excess is $E(B-V)=0.74 \pm 0.07 \mathrm{mag}$. Using a standard extinction curve from Fitzpatrick (1999) we thus obtained the dereddening parameters for each of our optical/NIR filters (Table 2).

Following Campana et al. (2004) and Burderi et al. (2003) we now attempt to account for the optical to NIR spectral energy distribution (corrected for interstellar absorption) with the simple model of an irradiated star with a blackbody spectrum (for the details of the modeling see Chakrabarty 1998, Eqs. (8) and (9)). We fit the data by using the irradiating luminosity $\left(L_{\text {irr }}\right)$ as free parameter and fixing the albedo of the $\operatorname{star}\left(\eta_{*}=0\right)$. We obtain an acceptable fit to all the data (see Fig. 5), with a reduced $\chi^{2}=1.3$ (4 degrees of freedom, null hypothesis probability of $28 \%$ ). The result of our fit is that the required irradiating luminosity is $4 \times 10^{33} \leq L_{\mathrm{irr}} \leq 5 \times 10^{33} \mathrm{erg} \mathrm{s}^{-1}$. Taking this value as a lower limit for the spin-down luminosity of a classical rotating magnetic dipole, we can estimate a neutron star's magnetic field greater than $6 \times 10^{7}$ Gauss. The presence of a disc is not required by our fit. In any case, we tried to fit our data with the model of an irradiated star plus a disc by using the irradiating luminosity and the internal disc radius $\left(R_{\text {in }}\right)$ as free parameters. We obtained an acceptable fit for similar values of the irradiating 


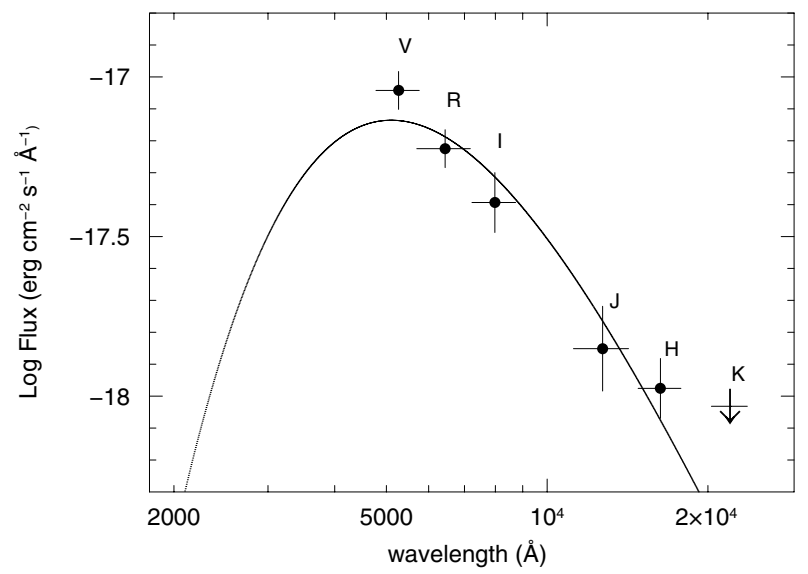

Fig. 5. Spectral energy distribution (optical to NIR) of IGR J00291+5934 during quiescence. Data are corrected for interstellar absorption. The plotted line represents the contribution of an irradiated star. See Sect. 5 for details.

luminosity $\left(L_{\mathrm{irr}} \sim 3 \times 10^{33} \mathrm{erg} \mathrm{s}^{-1}\right.$, reduced $\chi^{2}=1.8$ with 3 d.o.f., null hypothesis probability of $14 \%$ ), but the internal disc radius resulting from the fit is not much larger than neutron star $\left(R_{\text {in }} \leq 10^{6} \mathrm{~cm}\right)$. So, we cannot say much about the presence of a residual disc structure in the system during quiescence but, according to our results, it is not required to explain the observed optical/NIR quiescent emission.

The required irradiating luminosity is about two orders of magnitude larger than the observed quiescent X-ray luminosity. Such discrepancy is reminiscent of that observed for SAX J1808.4-3658 (Burderi et al. 2003; Campana et al. 2004), and can be explained with the presence in the system of a relativistic particle wind from an active pulsar which irradiates the companion star.

\section{Conclusions}

We have reported the detection of the optical and NIR counterpart of the accreting millisecond X-ray pulsar IGR J00291+5934 in quiescence. We provided precise astrometry and the results of multiband (VRIJHK) photometry. Photometry performed on $I$-band images and on a set of coadded $R$ and $I$ band frames, covering about $80-90 \%$ of the 2.46 h orbital period, shows variability of the source consistent with sinusoidal modulation at the orbital period with a semiamplitude of about $0.2-0.3 \mathrm{mag}$, suggesting the presence of an irradiated companion. From the observed $N_{\mathrm{H}}$, we estimate a color excess of $E(B-V)=0.74 \pm$ $0.07 \mathrm{mag}$. The observed optical/NIR quiescent luminosity, derived from our multiband photometry, can be modeled with an irradiated companion star providing a good agreement with the observed data. The presence of a residual disc component, even if possible, is not necessary for our model. The required irradiating luminosity is $4 \times 10^{33} \leq L_{\text {irr }} \leq 5 \times 10^{33} \mathrm{erg} \mathrm{s}^{-1}$, much larger than the observed X-ray luminosity in quiescence. If we assume this value as a lower limit for the spin-down luminosity, then the magnetic field of the neutron star results to be $>6 \times 10^{7}$ Gauss. As remarked for SAX J1808.4-3658 by Burderi et al. (2003) and Campana et al. (2004), the only source of energy available within the system that can supply such luminosity is the rotational energy of the neutron star emitted in the form of a relativistic particle wind. However, a direct detection of millisecond pulsations in the radio band for IGR J00291+5934 could be difficult for free-free absorption effects, due to the mass coming from the companion star and swept away by the radiation pressure of the pulsar. A search at high frequencies could be a solution to overcome this effect (see also Campana et al. 1998; Burderi et al. 2003; Campana et al. 2004).

Acknowledgements. We thank Vania Lorenzi, Giovanni Tessicini, Noemi Pinilla Alonso and Albar Garcia de Gurtubai Escuder for performing the observations at TNG. S.C. and P.D.A. acknowledge the Italian Space Agency for financial support through the project ASI I/R/023/05. P.D.A. acknowledge Daniele Malesani for useful discussion.

\section{References}

Alard, C. 2000, A\&AS, 144, 363

Alard, C., \& Lupton, R. 1998, ApJ, 503, 325

Baraffe, I., Chabrier, G., Allard, F., \& Hauschildt, P. H. 1998, A\&A, 337, 728

Bhattacharya, D., \& van den Heuvel, E. P. J. 1991, Phys. Rep., 2031

Bohlin, R. C., Savage, B. D., \& Drake 1979, ApJ, 224, 132

Burderi, L., Di Salvo, T., D’Antona, F., Robba, N. R., \& Testa, V. 2003, A\&A, 404, L43

Burderi, L., Di Salvo, T., Lavagetto, G., et al. 2006, ApJ, accepted [arXiv: astro-ph/0611222]

Campana, S. 2005, ATel, 535

Campana, S., Colpi, M., Mereghetti, S., Stella, L., \& Tavani, M. 1998, A\&AR, 8,279

Campana, S., Stella, L., Gastaldello, F., et al. 2002, ApJ, 575, L15

Campana, S., Ravasio, M., Israel, G. L., Mangano, V., \& Belloni, T. 2003, ApJ, 594, L39

Campana, S., D’Avanzo, P., Casares, J., et al. 2004, ApJ, 614, L49

Chakrabarty, D. 1998, ApJ, 492, 342

Chakrabarty, D., \& Morgan, E. H. 1998, Nature, 394, 346

Dickey, J. M., \& Lockman, F. J. 1990, ARA\&A, 28, 215

Eckert, D., Walter, R., Kretschmar, P., et al. 2004, ATel, 352

Falanga, M., Kuiper, L., \& Poutaten, J. 2005, A\&A, 444, 15

Fitzpatrick, E. L. 1999, PASP, 111, 63

Fender, R., De Bruyn, G., Pooley, G., \& Stappers, B. 2004, ATel, 361

Fox, D. B., \& Kulkarni, S. R. 2004, ATel, 354

Galloway, D. K., Chakrabarty, D., Morgan, E. H., \& Remillard, R. A. 2002, ApJ, 576, L137

Galloway, D. K., Markwardt, C. B., Morgan, E. H., Chakrabarty, D., \& Strohmayer, T. E. 2005, ApJ, 622, L45

Homer, L., Charles, P. A., Chakrabarty, D., \& van Zyl, L. 2001, MNRAS, 325, 1471

Jonker, P. G., Nelemans, G., Wang, Z., et al. 2003, MNRAS, 344, 201

Jonker, P. G., Campana, S., Steeghs, D., et al. 2005, MNRAS, 361, 511

Krauss, M. I., Wang, Z., Dullighan, A., et al. 2005, ApJ, 627, 910

Nowak, M. A., Paizis, A., Wilms, J., Ebisawa, K., et al. 2004, ATel, 369

Markwardt, C. B., \& Swank 2003, IAUC, 8144

Markwardt, C. B., Swank, J. H., Strohmayer, T. E., Zand, J. J. M. in't., \& Marshall, F. E. 2002, ApJ, 575, L21

Markwardt, C. B., Smith, E., \& Swank, J. H. 2003, IAUC, 8080

Markwardt, C. B., Swank, J. H., \& Strohmayer, T. E. 2004a, ATel, 353

Markwardt, C. B., Galloway, D. K., Chakrabarty, D., Morgan, E. H., \& Strohmayer, T. E. 2004b, ATel, 360

Monelli, M., Fiorentino, G., Burderi, L., et al. 2005, AIPC, 797, 565

Morgan, E., Kaaret, P., \& Vanderspek, R. 2005, ATel, 523

Paizis, A., Nowak, M. A., Wilms, J., et al. 2005, A\&A, 444, 357

Pooley, G. 2004, ATel, 355

Remillard, R. 2004, ATel, 357

Roelofs, G., Jonker, P. G., Steeghs, D., Torres, M., \& Nelemans, G. 2004, ATel, 356

Rupen, M. P., Dhawan, V., \& Mioduszewski, A. J. 2004, ATel, 364

Shaw, S. E., Mowlavi, N., Rodriguez, J., et al. 2005, A\&A, 432, L13

Strohmayer, T. E., \& Bildsten, L. 2005, in Compact Stellar X-ray Sources, ed. W. H. G. Lewin, \& M. van der Klis [arXiv: astro-ph/0301544]

Strohmayer, T. E., Markwardt, C. B., Swank, J. H., \& in't Zand, J. 2003, ApJ, 596, L67

Tauris, T. M., \& van den Heuvel, E. P. J. 2004, in Compact Stellar X-ray Sources, ed. W. H. G. Lewin, \& M. van der Klis (Cambridge: Cambridge University Press)

Torres, M. A. P., Jonker, P. G., Steeghs, D., et al. 2007 [arXiv: astro-ph/0701095]

Wijnands, R. 2005 [arXiv: astro-ph/0501264]

Wijnands, R., \& van der Klis, M. 1998, Nature, 394, 344

Wijnands, R., Guainazzi, M., van der Klis, M., \& Méndez, M. 2005, ApJ, 573, L45 